Bull. Korean Math. Soc. 48 (2011), No. 1, pp. 23-33

DOI 10.4134/BKMS.2011.48.1.023

\title{
On McCoy modules
}

\author{
Jian Cui and Jianlong Chen
}

\begin{abstract}
Extending the notion of McCoy rings, we introduce the class of McCoy modules. Over a given ring $R$, it contains the class of Armendariz modules (over $R$ ). Some properties of this class of modules are established, and equivalent conditions for McCoy modules are given. Moreover, we study the relationship between a module and its polynomial module. Several known results relating to McCoy rings can be obtained as corollaries of our results.
\end{abstract}

\section{Introduction}

All rings are associative with identity, and modules are unitary right modules. $R[x]$ denotes the polynomial ring over the ring $R$ and $M[x]$ denotes the polynomial module over the module $M$. For any subset $X$ of the module $M$, $r_{R}(X)$ stands for the right annihilator of $X$ in $R$.

McCoy proved in 1942 [15] that if two polynomials annihilate each other over a commutative ring, then each polynomial has a nonzero annihilator in the base ring. Rege and Chhawchharia [18] and Nielsen [16] independently introduced the notion of a McCoy ring. A ring $R$ is right McCoy if the equation $f(x) g(x)=0$ with $f(x) \in R[x]$ and $g(x) \in R[x] \backslash\{0\}$, implies that there exists a nonzero $r \in R$ such that $f(x) r=0$; left McCoy rings are defined similarly. A ring $R$ is called McCoy if it is both right and left McCoy. The class of McCoy rings contains the class of Armendariz rings (These rings are defined through the condition "whenever polynomials $f(x)=\sum_{i=0}^{m} a_{i} x^{i}, g(x)=\sum_{j=0}^{n} b_{j} x^{j} \in$ $R[x]$ satisfy $f(x) g(x)=0$, then $a_{i} b_{j}=0$ for every $i$ and $j$ '. See [18] for basic results on Armendariz rings). It is well-known that reduced rings (that is, rings without nonzero nilpotent elements) are Armendariz and therefore McCoy. Some properties of McCoy rings have been studied in [4], [9], [13], [16], [18], [19], [20] etc.

In this paper, we introduce the notion of a McCoy module. It is showed that semi-commutative modules over reduced rings, Bezout modules over right duo rings and projective modules over right McCoy rings are McCoy; if $R_{R}$ is

Received April 13, 2009; Revised February 8, 2010.

2010 Mathematics Subject Classification. Primary 16N60; Secondary 16P60.

Key words and phrases. McCoy module, McCoy ring, polynomial module, zip module. 
uniform (and $R$ is finitely cogenerated), then the class of McCoy $R$-modules is closed under direct sums (direct products); suppose that there exists the classical right quotient ring $Q$ of a ring $R$ and $M_{Q}$ is a module, then $M_{R}$ is McCoy if and only if $M_{Q}$ is McCoy; a module $M_{R}$ is McCoy if and only if its polynomial module $M[x]_{R[x]}$ is McCoy. We also provide an application of McCoy modules, and prove that if $M_{R}$ is McCoy, then $M$ is a zip $R$-module if and only if $M[x]$ is a zip $R[x]$-module. Consequently, several known results relating to McCoy rings can be obtained as corollaries of our results.

\section{McCoy modules}

According to Buhphang and Rege [3], a module $M_{R}$ is Armendariz if whenever $m(x)=\sum_{i=0}^{s} m_{i} x^{i} \in M[x]$ and $g(x)=\sum_{j=0}^{t} b_{j} x^{j} \in R[x]$ satisfy $m(x) g(x)$ $=0$, then $m_{i} b_{j}=0$ for every $i$ and $j$. The ring $R$ is Armendariz if and only if the module $R_{R}$ is Armendariz. Similarly, we define the following:

Definition 2.1. Let $M$ be a module over a ring $R$ and $M[x]$ be the corresponding polynomial module over $R[x] . M_{R}$ is called McCoy if $m(x) g(x)=0$, where $m(x)=\sum_{i=0}^{p} m_{i} x^{i} \in M[x]$ and $g(x)=\sum_{j=0}^{q} b_{j} x^{j} \in R[x] \backslash\{0\}$, implies that there exists a nonzero element $r \in R$ such that $m(x) r=0$.

Remark 2.2. (1) A ring $R$ is right McCoy if and only if the module $R_{R}$ is $\mathrm{McCoy}$;

(2) $M_{R}$ is McCoy if and only if, for all $m(x) \in M[x], r_{R[x]}(m(x)) \neq 0$ implies that $r_{R[x]}(m(x)) \bigcap R \neq 0$.

All Armendariz modules are obviously McCoy modules; the falsity of the converse was noted in [18, Remark 4.3] where it was pointed out that there exist commutative (and therefore McCoy) rings which are not Armendariz. See also [13, Theorem 2] and [11, Example 3] for a class of non-commutative examples. All torsion free modules over commutative domains are McCoy; modules over division rings are obviously McCoy. Several other examples will follow from our results later.

Proposition 2.3. (1) Every submodule of a McCoy module is McCoy. In particular, if $I$ is a right ideal of a right McCoy ring $R$, then $I_{R}$ is a McCoy module;

(2) $M$ is a McCoy module if and only if every finitely generated submodule of $M$ is $M c$ Coy;

(3) For any index set $\Gamma$, if $M_{i}$ is a McCoy $R_{i}$-module for each $i \in \Gamma$, then $\prod_{i \in \Gamma} M_{i}$ is a McCoy $\prod_{i \in \Gamma} R_{i}$-module;

(4) Let $I$ be any nonzero ideal of a ring $R$. Then $(R / I)_{R}$ is a McCoy module.

Proof. (1)-(3) are obvious. (4) For each $\overline{f(x)} \in(R / I)[x]$, take any nonzero $r \in I(\subseteq R)$. Then $f(x) r \in I[x]$, i.e., $\overline{f(x)} r=0$. 
In [3], Buhphang and Rege called a module $M_{R}$ is semi-commutative if $m a=0$ for $m \in M$ and $a \in R$, then $m R a=0$. We can infer that semicommutative modules need not be McCoy from Section 3 of [16]. But we have the following:

Proposition 2.4. Let $R$ be a reduced ring. Then a semi-commutative module $M_{R}$ is McCoy.

Proof. Suppose that $m(x)=m_{0}+m_{1} x+\cdots+m_{p} x^{p} \in M[x], g(x)=b_{0}+b_{1} x+$ $\cdots+b_{q} x^{q} \in R[x] \backslash\{0\}$ satisfy $m(x) g(x)=0$. We may assume that $b_{0} \neq 0$ (If not, set $g(x)=g^{\prime}(x) x^{k}$ with a minimal $k$ such that $b_{k} \neq 0$ since $g(x) \neq 0$, we have $\left.m(x) g^{\prime}(x)=0\right)$. This implies the following system of equations:

(0) $\quad m_{0} b_{0}=0$,

(1) $\quad m_{0} b_{1}+m_{1} b_{0}=0$,

(2) $\quad m_{0} b_{2}+m_{1} b_{1}+m_{2} b_{0}=0$,

$(p+q) \quad m_{p} b_{q}=0$.

Since $M_{R}$ is semi-commutative, we have $m_{0} b_{1} b_{0}=0$ from Eq. (0). Multiplying Eq. (1) on the right by $b_{0}$ yields $m_{1} b_{0}^{2}=0, \ldots$, multiplying Eq. $(k)$ on the right by $b_{0}^{k}$ yields $m_{k} b_{0}^{k+1}=0$ because $m_{l} b_{0}^{l+1}=0$ for $l=1, \ldots, k-1$. Since $R$ is reduced, $b_{0} \neq 0$ implies $b_{0}^{k} \neq 0$ for each $k$. Consequently, we obtain $m(x) b_{0}^{p+1}=0$.

The following example shows that the converse of Proposition 2.4 is not true.

Example 2.5. Let $R=\mathbb{Z}_{2}\langle a, b\rangle$ be the free associative algebra (with 1 ) over $\mathbb{Z}_{2}$ (the ring of integers modulo 2 ) generated by two indeterminates $a, b$. Let $I=\left\langle a^{2}\right\rangle$ be the ideal of $R$ generated by $a^{2}$. We take $M=R / I$. Clearly, $R$ is a reduced ring. By Proposition 2.3(4), $M_{R}$ is a McCoy module. However, $M_{R}$ is not semi-commutative. In fact: $\bar{a} a=0$ for $\bar{a} \in M$ and $a \in R$, but $a b a \notin I$, that is, $\bar{a} b a \neq 0$.

A ring is said to be right duo if all its right ideals are two-sided ideals.

Proposition 2.6. Every cyclic module over a right duo ring is McCoy.

Proof. In view of $[4$, Theorem 8.2], right duo rings are right McCoy. The result follows using Proposition 2.3(4).

We do not know whether the converse of Proposition 2.6 holds. Thus we have the following:

Question 1. Is $R$ a right duo ring in case every cyclic module over $R$ is McCoy?

Recall that a module is called a Bezout module if each of its finitely generated submodules is cyclic.

Proposition 2.7. Bezout modules over right duo rings are McCoy. 
Proof. Let $R$ be a right duo ring. By Proposition 2.6, every cyclic $R$-module is McCoy. Hence Bezout $R$-modules are McCoy by Proposition 2.3(2).

In the situation of the preceding proposition, the condition "the ring is right duo" is not superfluous and the converse is not true generally.

Example 2.8. (1) The condition that the ring $R$ is right duo is not superfluous. Let $D$ be a division ring, and $R=M_{n}(D)$ be an $n \times n$ matrix ring over $D$ for $n \geq 2$. Note that the ring $R$ is not right duo and $R_{R}$ is a semisimple module. Every submodule of $R_{R}$ being a direct summand, $R_{R}$ is a Bezout module. But $R_{R}$ is not McCoy by [4, Proposition 10.2] or [20, Theorem 2.1].

(2) The converse is not true since there are commutative domains which are not Bezout, for example the ring $\mathbb{Z}[x]$ of polynomials over the integers.

The classes of Armendariz modules and semi-commutative modules are closed under direct products as well as direct sums; this was noted by Buhphang and Rege in [3]. We ask:

Question 2. Under what conditions is the class of McCoy modules (over a given ring) closed under direct products and direct sums?

Next we record several results in the context of this question.

A module is called uniform if any two nonzero submodules have a nonzero intersection (see [6]). A module $M_{R}$ is said to be finitely cogenerated if for every set $\left\{A_{i} \mid i \in I\right\}$ of submodules $A_{i}$ of $M$ with $\bigcap_{i \in I} A_{i}=0$ there is a finite subset $\left\{A_{i} \mid i \in I_{0}\right\}$ (i.e., $I_{0} \subset I$ and $I_{0}$ is finite) with $\bigcap_{i \in I_{0}} A_{i}=0$ (see [2]).

Proposition 2.9. Let $\left\{M_{i}\right\}_{i \in \Lambda}$ be a family of McCoy $R$-modules for an index set $\Lambda$. Then we have:

(1) If $R_{R}$ is uniform, then a direct sum $M=\coprod_{i \in \Lambda} M_{i}$ is $M c$ Coy;

(2) If $\Lambda$ is an infinite set, $R_{R}$ is uniform and finitely cogenerated, then a direct product $M=\prod_{i \in \Lambda} M_{i}$ is McCoy.

Proof. We will use the same symbols in the proofs of (1) and (2). Let $m(x)=$ $\sum_{k=0}^{p}\left(m_{i k}\right)_{i \in \Lambda} x^{k} \in M[x], g(x) \in R[x] \backslash\{0\}$ satisfy $m(x) g(x)=0$. Let $m_{i}(x)=$ $\sum_{k=0}^{p} m_{i k} x^{k} \in M_{i}[x]$. Since $M_{i}$ is McCoy and $m_{i}(x) g(x)=0$, there exists a nonzero $r_{i} \in R$ such that $m_{i}(x) r_{i}=0$.

To prove (1), note that the set $\Lambda^{\prime}=\left\{i \in \Lambda \mid m_{i}(x) \neq 0\right\}$ is a finite set. Put $U=\bigcap_{i \in \Lambda^{\prime}} r_{i} R$. Since $R_{R}$ is uniform, we have $U \neq 0$. Take any $r \in U \backslash\{0\}$, then $m_{i}(x) r=0$ for each $i$, whence $m(x) r=0$. Thus, $M=\coprod_{i \in \Lambda} M_{i}$ is McCoy.

To show (2), $R_{R}$ is uniform implies that $\bigcap_{i \in \Lambda_{0}} r_{i} R \neq 0$ for any finite subset $\Lambda_{0} \subset \Lambda$, and since $R_{R}$ is finitely cogenerated, it follows that $U=\bigcap_{i \in \Lambda} r_{i} R \neq 0$. Similar to the proof of (1), we are done.

Proposition 2.10. Let $M$ be a McCoy R-module. Then a direct sum of copies of $M$ is McCoy. 
Proof. For an index set $I$, let $M^{(I)}$ be a direct sum of copies of $M$. By Proposition 2.3(2), we can assume $I$ to be finite. We prove the result in three steps.

Step 1. We show that $M^{(2)}$ is McCoy. Let $m(x)=\sum_{k=0}^{p}\left(m_{i k}\right)_{i \in\{0,1\}} x^{k} \in$ $M^{(2)}[x]$ and $g(x) \in R[x] \backslash\{0\}$ satisfy $m(x) g(x)=0$. Let $m_{i}(x)=\sum_{k=0}^{p} m_{i k} x^{k} \in$ $M[x]$, where $i=0,1$. Write $u=2 p+q$, where $q$ is the degree of $g(x)$. Let $m^{\prime}(x)=m_{0}(x)+m_{1}(x) x^{u}$; then $m^{\prime}(x) g(x)=0$. Since $M_{R}$ is McCoy, there exists a nonzero $r \in R$ such that $m^{\prime}(x) r=0$, that is $m_{i k} r=0$ for all $i$ and $k$. Therefore $m(x) r=0$.

Step 2. Write $m=2^{n}$ for any $n \geq 1$. By Step $1, M^{(m)}$, the direct sum of $m$ copies of $M$, is McCoy.

Step 3. For every natural number $k$ the module $M^{(k)}$ is a submodule of $M^{\left(2^{n}\right)}$ for some $n$, and so the result holds for $M^{(k)}$ by Proposition 2.3(1).

Corollary 2.11. Projective modules over right McCoy rings are McCoy.

Proof. It is well-known that every projective module is a direct summand of a free module. Let $R$ be a right McCoy ring. Then $R^{(I)}$ is a McCoy $R$-module for every indexing set $I$ by Proposition 2.10. So the result follows from Proposition $2.3(1)$.

In what follows $R_{n}$ denotes (for a positive integer $n$ ) the following subring of the ring of all matrices over $R$ :

$$
R_{n}=\left\{\left(\begin{array}{cccc}
a & a_{12} & \ldots & a_{1 n} \\
0 & a & \ldots & a_{2 n} \\
\vdots & \vdots & \ddots & \vdots \\
0 & 0 & \ldots & a
\end{array}\right): a, a_{i j} \in R\right\}
$$

we also consider the following subgroup of the additive group of all formal matrices over $M$, namely,

$$
M_{n}=\left\{\left(\begin{array}{cccc}
m & m_{12} & \ldots & m_{1 n} \\
0 & m & \ldots & m_{2 n} \\
\vdots & \vdots & \ddots & \vdots \\
0 & 0 & \ldots & m
\end{array}\right): m, m_{i j} \in M\right\} .
$$

Then $M_{n}$ is an $R_{n}$-module under the usual matrix addition operation and the following scalar product operation. For $W=\left(w_{i j}\right) \in M_{n}$ and $A=\left(a_{i j}\right) \in R_{n}$, $W A=\left(m_{i j}\right)$ with $m_{i j}=\sum_{k=1}^{n} w_{i k} a_{k j}$ for $i, j=1,2, \ldots, n$.

We have the following result:

Proposition 2.12. $M$ is a McCoy R-module if and only if $M_{n}$ is a McCoy $R_{n}$-module.

Proof. The proof is similar to that of [13, Theorem 2].

" $\Rightarrow$ ". Every $\alpha(x) \in M_{n}[x]$ (resp., $\beta(x) \in R_{n}[x]$ ) can be written as the form of a matrix. Write $\alpha_{i j}(x)=[\alpha(x)]_{i, j}\left(\right.$ resp., $\left.\beta_{i j}(x)=[\beta(x)]_{i, j}\right)$ the $(i, j)$-entry of $\alpha(x)$ (resp., $\beta(x)$ ). Let $E_{i j}$ denote matrix units. 
Suppose that $\alpha(x) \beta(x)=0$, where $\alpha(x) \in M_{n}[x]$ and $\beta(x) \in R_{n}[x] \backslash\{0\}$. We show that there exists a nonzero $A \in R_{n}$ such that $\alpha(x) A=0$. Now we proceed with the following cases.

Case 1. If $\alpha_{11}(x) \neq 0, \beta_{11}(x) \neq 0$, then $\alpha_{11}(x) \beta_{11}(x)=0$, where $\alpha_{11}(x)=$ $[\alpha(x)]_{1,1}, \beta_{11}(x)=[\beta(x)]_{1,1}$. Since $M_{R}$ is McCoy, there exists $r \in R \backslash\{0\}$ such that $\alpha_{11}(x) r=0$. Put $A=r E_{1 n}$, then $\alpha(x) A=0$.

Case 2. If $\alpha_{11}(x) \neq 0, \beta_{11}(x)=0$, then there exists a nonzero entry $\beta_{k l}(x)$ such that all entries of the matrix to the left of and below it vanish since $\beta(x) \neq 0$. So $\alpha_{11}(x) \beta_{k l}(x)=0$. Hence there exists $r \in R \backslash\{0\}$ such that $\alpha_{11}(x) r=0$. Write $A=r E_{1 n}$, then $\alpha(x) A=0$.

Case 3. If $\alpha_{11}(x)=0$, then for any $r \in R \backslash\{0\}, \alpha(x) A=0$ with $A=r E_{1 n}$. This completes the proof of this implication.

" $\Leftarrow "$. Assume that $m(x) g(x)=0$, where $m(x) \in M[x], g(x) \in R[x] \backslash\{0\}$. Let $\alpha(x)=m(x) I_{n}, \beta(x)=g(x) I_{n}$, where $I_{n}$ is the $n \times n$ identity matrix. Then $\alpha(x) \in M_{n}[x], \beta(x) \in R_{n}[x] \backslash\{0\}$ and $\alpha(x) \beta(x)=0$. Since $M_{n}$ is a McCoy $R_{n^{-}}$ module, there exists a nonzero $A \in R_{n}$ such that $\alpha(x) A=0$. Obviously, there exists $r \in R \backslash\{0\}$ such that $m(x) r=0$. Therefore, $M_{R}$ is McCoy.

Corollary 2.13 ([13, Theorem 2]). A ring $R$ is McCoy if and only if the ring $R_{n}$ is $M c$ Coy.

For a commutative domain $R$ and a module $M_{R}$, the torsion submodule of $M_{R}$ is defined by $T(M)=\left\{x \in M \mid r_{R}(x) \neq 0\right\}$. We have the following result.

Proposition 2.14. Let $D$ be a commutative domain and $M$ be a D-module. The module $M_{D}$ is McCoy if and only if its torsion submodule $T(M)$ is McCoy.

Proof. Let $m(x)=\sum_{i=0}^{p} m_{i} x^{i} \in M[x], g(x)=\sum_{j=0}^{q} d_{j} x^{j} \in D[x] \backslash\{0\}$ satisfy $m(x) g(x)=0$. We have the system of equations $m_{0} d_{0}=0, m_{0} d_{1}+m_{1} d_{0}=$ $0, m_{0} d_{2}+m_{1} d_{1}+m_{2} d_{0}=0, \ldots, m_{p} d_{q}=0$. We may assume $d_{0} \neq 0$ since $g(x) \neq 0$. Now multiplying $m_{0} d_{1}+m_{1} d_{0}=0$ by $d_{0}$ on the right yields $m_{1} d_{0}^{2}=0$, thus $d_{0}^{2}$ annihilates both $m_{0}$ and $m_{1}$; similarly, $m_{0} d_{2}+m_{1} d_{1}+m_{2} d_{0}=0$ and multiplying on the right by $d_{0}^{2}$ yields $m_{2} d_{0}^{3}=0$. Continuing this process, we have $m_{i} d_{0}^{i+1}=0$ for $i=3, \ldots, p$. So $m_{i} \in T(M)$, i.e., $m(x) \in T(M)[x]$. Since $T(M)$ is McCoy as a $D$-module, we conclude that there exists a nonzero $d \in D$ such that $m_{i} d=0$ for all $i$. Hence, $m(x) d=0$. The other implication is trivial.

Proposition 2.15. Let $R$ be a commutative principal ideal ring. Then every $R$-module is McCoy. In particular, every $\mathbb{Z}$-module is McCoy.

Proof. By Proposition 2.3(2) we can assume $M$ to be finitely generated. Since $R$ is a commutative principal ideal ring, it is arithmetical (i.e., is a ring in which every finitely generated ideal is locally principal). By [3, Proposition 3.8], $M_{R}$ is Armendariz, and thus is McCoy.

A module $M_{R}$ is called reduced if for any $m \in M$ and $a \in R, m a=0$ implies $m R \cap M a=0$; and reduced modules are Armendariz by [12, Lemma 1.5]. 
Remark 2.16. It is shown in [17, Theorem 2.16] that over strongly regular rings (i.e., over rings satisfying 'for every $a \in R$, there exists $b \in R$ such that $a=b a^{2}$ ) all modules are reduced. It follows that over such rings all modules are Armendariz and are therefore McCoy.

A classical right quotient ring for $R$ is a $\operatorname{ring} Q$ which contains $R$ as a subring in such a way that every regular element (i.e., non-zero-divisor) of $R$ is invertible in $Q$ and $Q=\left\{a \mu^{-1}: a, \mu \in R, \mu\right.$ regular $\}$. The free algebra $L\langle x, y\rangle$ in two indeterminates over a field $L$ is a well-known example of a domain which does not have a classical right quotient ring.

Theorem 2.17. Suppose that there exists the classical right quotient ring $Q$ of a ring $R$ and $M$ is a $Q$-module. Then $M_{R}$ is $M c C o y$ if and only if $M_{Q}$ is McCoy.

Proof. Suppose that $M_{R}$ is a McCoy module. Let $m(x)=\sum_{i=0}^{p} m_{i} x^{i} \in M[x]$ and $g(x)=\sum_{j=0}^{q} b_{j} x^{j} \in Q[x] \backslash\{0\}$ with $m(x) g(x)=0$. Since $Q$ is the classical right quotient ring of $R$, by [14, Proposition 2.1.16], we may assume that $b_{j}=$ $b_{j}^{\prime} \mu^{-1}$ with $b_{j}^{\prime} \in R$ and some regular element $\mu \in R$. Write $g^{\prime}(x)=\sum_{j=0}^{q} b_{j}^{\prime} x^{j}$. Then $g^{\prime}(x) \in R[x] \backslash\{0\}$ and $0=m(x) g(x)=\sum_{i=0}^{p} \sum_{j=0}^{q} m_{i} b_{j}^{\prime} x^{i+j} \mu^{-1}=$ $m(x) g^{\prime}(x) \mu^{-1}$. So we have $m(x) g^{\prime}(x)=0$. Since $M_{R}$ is McCoy, there exists $r \in R \backslash\{0\}(\subseteq Q \backslash\{0\})$ such that $m(x) r=0$.

Conversely, let $n(x)=\sum_{i=0}^{p} n_{i} x^{i} \in M[x]$ and $h(x)=\sum_{j=0}^{q} c_{j} x^{j} \in R[x] \backslash\{0\}$ $(\subseteq Q[x] \backslash\{0\})$ satisfy $n(x) h(x)=0$. Since $M_{Q}$ is McCoy, there exists a nonzero element $d \in Q$ such that $n_{i} d=0$ for $i=0,1, \ldots, p$. Because $Q$ is the classical right quotient ring of $R$, we have $d=a \mu^{-1}$ for some nonzero $a \in R$ and regular element $\mu$. Then $n_{i} a=n_{i} d \mu=0$. Therefore $M_{R}$ is a McCoy module.

\section{Polynomial modules}

In this section, we study the relations between an $R$-module $M$ and the polynomial module $M[x]$ over $M$. Moreover, we will give an application of McCoy modules.

Let $M$ be an $R$-module. If $S$ is a multiplicatively closed subset of central regular elements of $R$, then $S^{-1} M$ has an $S^{-1} R$-module structure. $M$ is called $S$-torsion free if whenever $m$ is a nonzero element of $M$ and $s$ is an element of $S$, we have $m s \neq 0$.

Lemma 3.1. Let $M$ be $S$-torsion free. Then the $R$-module $M$ is McCoy if and only if the $S^{-1} R$-module $S^{-1} M$ is McCoy.

Proof. Suppose that $M_{R}$ is a McCoy module. Let $m^{\prime}(x)=\sum_{i=0}^{p} \frac{m_{i}}{s} x^{i} \in$ $S^{-1} M[x]$ and $g^{\prime}(x)=\sum_{j=0}^{q} \frac{b_{j}}{t} x^{j} \in S^{-1} R[x] \backslash\{0\}$ satisfy $m^{\prime}(x) g^{\prime}(x)=0$. It is easily deduced (using $M$ is $S$-torsion free) that $m(x) g(x)=0$, where $m(x)=$ $\sum_{i=0}^{p} m_{i} x^{i} \in M[x], g(x)=\sum_{j=0}^{q} b_{j} x^{j} \in R[x] \backslash\{0\}$. Since $M$ is McCoy, there exists a nonzero element $r \in R$ such that $m(x) r=0$, yielding $m^{\prime}(x) r=0$; 
clearly, $r$ is a nonzero element of $S^{-1} R$ as well. Hence $S^{-1} M$ is McCoy as an $S^{-1} R$-module.

Conversely, let $n(x)=\sum_{i=0}^{p} n_{i} x^{i} \in M[x], h(x)=\sum_{j=0}^{q} c_{j} x^{j} \in R[x] \backslash\{0\}$. Suppose that $n(x) h(x)=0$. Since $S^{-1} M$ is McCoy, there exists $\frac{r}{t} \in S^{-1} R \backslash\{0\}$ such that $n(x) \frac{r}{t}=0$. It follows that $n(x) r=0$. Thus $M_{R}$ is McCoy.

We use $R\left[x ; x^{-1}\right]$ to denote the Laurent polynomial ring over $R$. For a module $M_{R}$, let $M\left[x ; x^{-1}\right]=\left\{\sum_{i=k}^{n} m_{i} x^{i}: k, n \in \mathbb{Z}, m_{i} \in M\right\}$. As in Lee and Zhou [12], $M\left[x ; x^{-1}\right]$ is an $R\left[x ; x^{-1}\right]$-module under the obvious addition operation and the following scalar product operation. For $m(x)=\sum_{i} m_{i} x^{i} \in M\left[x ; x^{-1}\right]$ and $f(x)=\sum_{j} a_{j} x^{j} \in R\left[x ; x^{-1}\right]$, then $m(x) f(x)=\sum_{k}\left(\sum_{i+j=k} m_{i} a_{j}\right) x^{k}$.

Theorem 3.2. For an $R$-module $M$, the following statements are equivalent:

(1) $M$ is a McCoy $R$-module;

(2) $M[x]$ is a McCoy $R[x]$-module;

(3) $M\left[x ; x^{-1}\right]$ is a McCoy $R\left[x ; x^{-1}\right]$-module.

Proof. $(1) \Rightarrow(2)$ Suppose that $M$ is a McCoy module. Let $n(y) \in M[x][y]$ and $h(y) \in R[x][y] \backslash\{0\}$ with $n(y) h(y)=0$. Write $n(y)=n_{0}(x)+n_{1}(x) y+$ $\cdots+n_{p}(x) y^{p}$ and $h(y)=h_{0}(x)+h_{1}(x) y+\cdots+h_{q}(x) y^{q}$, where $n_{i}(x)=$ $\sum_{k=0}^{p_{i}} n_{i k} x^{k} \in M[x]$ and $h_{j}(x)=\sum_{l=0}^{q_{j}} c_{j l} x^{l} \in R[x]$. As in the proof of $\left[1\right.$, Theorem 2], we let $u=\sum_{i=0}^{p} \operatorname{deg}\left(n_{i}(x)\right)+\sum_{j=0}^{q} \operatorname{deg}\left(h_{j}(x)\right)$, where the degree of $n_{i}(x)$ is as polynomial in $M[x]$, the degree of $h_{j}(x)$ is as polynomial in $R[x]$ and the degree of the zero polynomial is taken to be zero. Then $n\left(x^{u}\right)=\sum_{i=0}^{p} n_{i}(x) x^{u i} \in M[x], h\left(x^{u}\right)=\sum_{j=0}^{q} h_{j}(x) x^{u j} \in R[x]$, and the set of coefficients of $n_{i}(x)$ 's (resp., $h_{j}(x)$ 's) equals the set of coefficients of $n\left(x^{u}\right)$ (resp., $\left.h\left(x^{u}\right)\right)$. Since $n(y) h(y)=0, x$ commutes with the elements of $R, n\left(x^{u}\right) h\left(x^{u}\right)=0$. By hypothesis, there exists $r \in R \backslash\{0\}$ such that $n\left(x^{u}\right) r=0$. Thus $n(y) r=0$.

$(2) \Rightarrow(1)$ Assume that $M[x]$ is McCoy, and let $m(x)=\sum_{i=0}^{p} m_{i} x^{i} \in M[x]$, $g(x)=\sum_{j=0}^{q} b_{j} x^{j} \in R[x] \backslash\{0\}$ satisfy $m(x) g(x)=0$. Set $m(y)=\sum_{i=0}^{p} m_{i} y^{i}$ and $g(y)=\sum_{j=0}^{q} b_{j} y^{j}$. Then $m(y) \in M[x][y], g(y) \in R[x][y] \backslash\{0\}$ and $m(y) g(y)=$ 0 . Since $M[x]_{R[x]}$ is McCoy, there exists a nonzero $g^{\prime}(x) \in R[x]$ such that $m(y) g^{\prime}(x)=0$. Let $c$ be a nonzero coefficient of $g^{\prime}(x)$. Then $m(y) c=0$. Clearly, $m(x) c=0$. So $M_{R}$ is a McCoy module.

$(2) \Leftrightarrow(3)$ Let $S=\left\{1, x, x^{2}, \ldots\right\}$. Then $S$ is a multiplicatively closed subset of central regular elements of $R[x]$. Since $M\left[x ; x^{-1}\right]=S^{-1} M[x]$ and $R\left[x ; x^{-1}\right]=$ $S^{-1} R[x]$, by Lemma 3.1 , we are done.

Remark 3.3. From the results of Section 3 of [4], we can obtain that the module $M[[x]]_{R[[x]]}$ may not be McCoy even if $M_{R}$ is a McCoy module, where the definition of the module $M[[x]]_{R[[x]]}$ is similar to that of the module $M\left[x ; x^{-1}\right]_{R\left[x ; x^{-1}\right]}$.

We write $M_{n}(R)$ for the $n \times n$ matrix ring over $R$. For a module $M_{R}$ and $A=\left(a_{i j}\right) \in M_{n}(R)$, let $M A=\left\{\left(m a_{i j}\right): m \in M\right\}$. For $n \geq 2$, let $V=$ 
$\sum_{i=1}^{n-1} E_{i(i+1)}$ where $\left\{E_{i j}: 1 \leq i, j \leq n\right\}$ are the matrix units, and set $V_{n}(R)=$ $R I_{n}+R V+\cdots+R V^{n-1}$ and $V_{n}(M)=M I_{n}+M V+\cdots+M V^{n-1}$. Then $V_{n}(R)$ is a ring and $V_{n}(M)$ becomes a right module over $V_{n}(R)$ under usual addition and multiplication of matrices. There is a ring isomorphism $\theta: V_{n}(R) \rightarrow R[x] /\left(x^{n}\right)$ given by $\theta\left(r_{0} I_{n}+r_{1} V+\cdots+r_{n-1} V^{n-1}\right)=r_{0}+r_{1} x+\cdots+r_{n-1} x^{n-1}+\left(x^{n}\right)$, and an Abelian group isomorphism $\phi: V_{n}(M) \rightarrow M[x] /\left(M[x]\left(x^{n}\right)\right)$ given by $\phi\left(m_{0} I_{n}+m_{1} V+\cdots+m_{n-1} V^{n-1}\right)=m_{0}+m_{1} x+\cdots+m_{n-1} x^{n-1}+M[x]\left(x^{n}\right)$ such that $\phi(W A)=\phi(W) \theta(A)$ for all $W \in V_{n}(M)$ and $A \in V_{n}(R)$.

Proposition 3.4. $M$ is a McCoy R-module if and only if $M[x] / M[x]\left(x^{n}\right)$ is a McCoy $R[x] / R[x]\left(x^{n}\right)$-module for any integer $n \geq 2$.

Proof. By the remark above, it suffices to show that $M_{R}$ is McCoy if and only if $V_{n}(M)_{V_{n}(R)}$ is McCoy.

" $\Rightarrow$ ". Suppose that $W(x) A(x)=0$ where $W(x)=\sum_{i=0}^{p} W_{i} x^{i} \in V_{n}(M)[x]$ and $A(x)=\sum_{j=0}^{q} A_{j} x^{j} \in V_{n}(R)[x] \backslash\{0\}$. Write $W_{i}=m_{i 0} I_{n}+m_{i 1} V+\cdots+$ $m_{i(n-1)} V^{n-1}$ and $A_{j}=a_{j 0} I_{n}+a_{j 1} V+\cdots+a_{j(n-1)} V^{n-1}$ for $0 \leq i \leq p$ and $0 \leq j \leq q$. It follows from $W(x) A(x)=0$ that $\left[m_{0}(x) I_{n}+m_{1}(x) V+\cdots+\right.$ $\left.m_{n-1}(x) V^{n-1}\right]\left[a_{0}(x) I_{n}+a_{1}(x) V+\cdots+a_{n-1}(x) V^{n-1}\right]=0$, where $m_{k}(x)=$ $m_{0 k}+m_{1 k} x+\cdots+m_{p k} x^{p} \in M[x]$ and $a_{l}(x)=a_{0 l}+a_{1 l} x+\cdots+a_{q l} x^{q} \in R[x]$ for $0 \leq k, l \leq n-1$, and hence $\sum_{k+l=t} m_{k}(x) a_{l}(x)=0$ for $t=0,1, \ldots, n-1$. In particular, we have

$$
m_{0}(x) a_{l_{0}}(x)=0
$$

with a minimal $l_{0}$ such that $a_{l_{0}}(x) \neq 0$ (Such a $l_{0}$ exists since $\left.A(x) \neq 0\right)$. Since $M_{R}$ is McCoy, there exists a nonzero $r \in R$ such that $m_{0}(x) r=0$. Let $A=r E_{1 n}$. Then $A \in V_{n}(R) \backslash\{0\}$ and $W(x) A=0$. So $V_{n}(M)_{V_{n}(R)}$ is McCoy.

" $\Leftarrow$ ". The proof of this implication is similar to that of in the "if" part of Proposition 2.12 .

Corollary 3.5 ([20, Theorem 2.3]). For a ring $R$, the following statements are equivalent:

(1) $R$ is a right $M c C o y$ ring;

(2) $R[x]$ is a right McCoy ring;

(3) $R\left[x ; x^{-1}\right]$ is a right McCoy ring;

(4) $R[x] /\left(x^{n}\right)$ is a right McCoy ring.

The following definition is due to Zhang and Chen [22]. A module $M_{R}$ is a zip module if for any subset $X$ of $M, r_{R}(X)=0$ implies $r_{R}(Y)=0$ for some finite subset $Y$ of $X$. By [5, Proposition 1] and [10, Example 10], (in general) the class of McCoy modules neither contains nor is contained in the class of zip modules. Even if $R_{R}$ is a zip module, $R[x]_{R[x]}$ need not be zip by [5, Example 2] (Some notable results on zip rings have appeared in [7], [8], [21], etc).

Theorem 3.6. Let $M$ be a McCoy R-module. Then $M$ is a zip R-module if and only if $M[x]$ is a zip $R[x]$-module. 
Proof. Suppose that $M[x]$ is zip. Let $Y \subseteq M$ with $r_{R}(Y)=0$. If $f(x)=$ $a_{0}+a_{1} x+\cdots+a_{n} x^{n} \in r_{R[x]}(Y)$, then $m f(x)=0$ for any $m \in Y$. Thus $m a_{i}=0$, and so $a_{i} \in r_{R}(Y)=0, i=1,2, \ldots, n$. Therefore $f(x)=0$, i.e., $r_{R[x]}(Y)=0$. Since $M[x]$ is zip, there exists a finite subset $Y_{0} \subseteq Y$ such that $r_{R[x]}\left(Y_{0}\right)=0$. Hence, $r_{R}\left(Y_{0}\right)=r_{R[x]}\left(Y_{0}\right) \bigcap R=0$.

Conversely, suppose that $M$ is zip. Let $X \subseteq M[x]$ with $r_{R[x]}(X)=0$. Now let $Y$ be the set of all coefficients of elements in $X$. Then $Y \subseteq M$. If $a \in r_{R}(Y)$, then $w a=0$ for each $w \in Y$. Thus $m(x) a=0$ for any $m(x) \in X$, and so $a \in r_{R[x]}(X)=0$. That is $r_{R}(Y)=0$. Since $M$ is zip, there exists a finite subset $Y_{0}=\left\{w_{1}, w_{2}, \ldots, w_{t}\right\} \subseteq Y$ such that $r_{R}\left(Y_{0}\right)=0$. For each $w_{i} \in Y_{0}$ and $i=1,2, \ldots, t$, let $m_{w_{i}}(x) \in X$ be such that some coefficient of $m_{w_{i}}(x)$ is $w_{i}$. Let $X_{0}=\left\{m_{w_{1}}(x), m_{w_{2}}(x), \ldots, m_{w_{t}}(x)\right\} \subseteq X$ and $Y_{1}$ be the set of all coefficients of elements in $X_{0}$, where $m_{w_{i}}(x)=\sum_{l=0}^{p_{w_{i}}} a_{w_{i} l} x^{l}$. Then $Y_{0} \subseteq Y_{1}$ and so $r_{R}\left(Y_{1}\right) \subseteq$ $r_{R}\left(Y_{0}\right)=0$. If $f(x)=\sum_{j=0}^{n} b_{j} x^{j} \in r_{R[x]}\left(X_{0}\right) \backslash\{0\}$, then $m_{w_{i}}(x) f(x)=0$ for $i=1,2, \ldots, t$. Write $u=\sum_{k=1}^{t} p_{w_{k}}+n$, and $n(x)=\sum_{i=1}^{t} m_{w_{i}}(x) x^{u i}(\in M[x])$, we have $n(x) f(x)=0$. Since $M_{R}$ is McCoy, there exists $r \in R \backslash\{0\}$ such that $n(x) r=0$, that is $a_{w_{i} l} r=0$ for each $w_{i}$ and $l$. So $r \in r_{R}\left(Y_{1}\right)=0$. This is a contradiction. Therefore $f(x)=0$, that is, $r_{R[x]}\left(X_{0}\right)=0$.

Corollary 3.7. Let $R$ be a right McCoy ring. Then $R$ is right zip if and only if $R[x]$ is right zip.

Corollary 3.8 ([22, Theorem 2.2]). Let $M$ be an Armendariz R-module. Then $M$ is a zip $R$-module if and only if $M[x]$ is a zip $R[x]$-module.

Corollary 3.9 ([10, Theorem 11]). Let $R$ be an Armendariz ring. Then $R$ is right zip if and only if $R[x]$ is right zip.

Acknowledgments. The authors are highly grateful to the referee for correcting many errors and providing some new proofs and examples which led to substantial improvements of the paper. In particular, Proposition 2.6 and Example $2.8(2)$ were suggested to the authors by the referee. This research is supported by the National Natural Science Foundation of China (10871042, 10971024), and the Specialized Research Fund for the Doctoral Program of Higher Education (200802860024).

\section{References}

[1] D. D. Anderson and V. Camillo, Armendariz rings and Gaussian rings, Comm. Algebra 26 (1998), 2265-2272.

[2] F. R. Anderson and K. R. Fuller, Rings and Categories of Modules, Second edition. Graduate Texts in Mathematics, 13. Springer-Verlag, New York, 1992.

[3] A. M. Buhphang and M. B. Rege, Semi-commutative modules and Armendariz modules, Arab. J. Math. Sci. 8 (2002), no. 1, 53-65.

[4] V. Camillo and P. P. Nielsen, McCoy rings and zero-divisors, J. Pure Appl. Algebra 212 (2008), no. 3, 599-615.

[5] F. Cedó, Zip rings and Mal'cev domains, Comm. Algebra 19 (1991), 1983-1991. 
[6] C. Faith, Algebra I, Rings, Modules and Categories, 205-207, Springer-Verlag, New York, 1981.

[7] _ Rings with zero intersection property on annihilators: Zip rings, Publ. Mat. 33 (1989), no. 3, 329-332

[8] _ Annihilator ideals, associated primes and Kasch-McCoy commutative rings, Comm. Algebra 19 (1991), no. 7, 1867-1892.

[9] A. Forsythe, Divisors of zero in polynomial rings, Amer. Math. Monthly 50 (1943), 7-8.

[10] C. Y. Hong, N. K. Kim, T. K. Kwak, and Y. Lee, Extensions of zip rings, J. Pure Appl. Algebra 195 (2005), no. 3, 231-242.

[11] N. K. Kim and Y. Lee, Armendariz rings and reduced rings, J. Algebra 223 (2000), no. $2,477-488$.

[12] T. K. Lee and Y. Q. Zhou, Reduced modules, Rings, modules, algebras, and abelian groups, 365-377, Lecture Notes in Pure and Appl. Math., 236, Dekker, New York, 2004.

[13] Z. Lei, J. L. Chen, and Z. L. Ying, A question on McCoy rings, Bull. Austral. Math. Soc. 76 (2007), no. 1, 137-141.

[14] J. C. McConnell and J. C. Robson, Noncommutative Noetherian Rings, Wiley, New York, 1987.

[15] N. H. McCoy, Remarks on divisors of zero, Amer. Math. Monthly 49 (1942), 286-295.

[16] P. P. Nielsen, Semi-commutativity and the McCoy condition, J. Algebra 298 (2006), no. $1,134-141$.

[17] M. B. Rege and A. M. Buhphang, On reduced modules and rings, Int. Electron. J. Algebra 3 (2008), 58-74.

[18] M. B. Rege and S. Chhawchharia, Armendariz rings, Proc. Japan Acad. Ser. A Math. Sci. 73 (1997), no. 1, 14-17.

[19] W. R. Scott, Divisors of zero in polynomial rings, Amer. Math. Monthly 61 (1954), 336.

[20] Z. L. Ying, J. L. Chen, and Z. Lei, Extensions of McCoy rings, Northeast Math. J. 24 (2008), no. 1, 85-94.

[21] J. M. Zelmanowitz, The finite intersection property on annihilator right ideals, Proc. Amer. Math. Soc. 57 (1976), no. 2, 213-216.

[22] C. P. Zhang and J. L. Chen, Zip modules, Northeast Math. J. 24 (2008), no. 3, 240-256.

JiAN CUI

Department of Mathematics

SOUTHEAST UNIVERSITY

NANJing 210096, P. R. China

E-mail address: jcui126@126.com

JiANLONG CHEN

Department of Mathematics

SOUTHEAST UNIVERSITY

NANJING 210096, P. R. ChINA

E-mail address: jlchen@seu.edu.cn 